\title{
STANDBY REDUNDANCY ALLOCATIONS IN SERIES AND PARALLEL SYSTEMS
}

\author{
NEERAJ MISRA, ${ }^{* * *}$ \\ AMIT KUMAR MISRA **** AND \\ ISHWARI DUTT DHARIYAL, ${ }^{* * * * *}$ Indian Institute of Technology Kanpur
}

\begin{abstract}
To enhance the performance of a system, a common practice employed by reliability engineers is to use redundant components in the system. In this paper we compare lifetimes of series (parallel) systems arising out of different allocations of one or two standby redundancies. These comparisons are made with respect to the increasing concave (convex) order, the hazard rate order, and the stochastic precedence order. The main results extend some related conclusions in the literature.
\end{abstract}

Keywords: Hazard rate order; IFR; increasing concave (convex) order; likelihood ratio order; reversed hazard rate order; stochastic precedence order; usual stochastic order

2010 Mathematics Subject Classification: Primary 62N05

Secondary 90B25

\section{Introduction}

The problem of where and how to allocate redundant components in a system, in order to optimize its lifetime or some other performance characteristic, is interesting and important in reliability theory and its applications. It has posed many interesting theoretical problems in probability, which have attracted the attention of many researchers (see [2], [9], [10], and [12], among others).

Generally, there are two methods to allocate redundant components in a system: the active (or parallel) redundancy allocation, and the standby redundancy allocation. Parallel redundancy is used when replacement of components during the operation of the system is not possible. In this case redundant components are connected in parallel with the components of the system and function simultaneously with them (which leads to the consideration of the maximum of random variables). Standby redundancy is used when replacement of components during the operation of the system is possible. In this case a spare starts functioning immediately after the failure of the corresponding component in the system (which leads to the consideration of the convolution of random variables). Evidently, to achieve the desired system reliability, the standby redundancy is more economical than the parallel redundancy. Performances of various allocations can be compared through stochastic comparisons between the corresponding system lifetimes.

Let $X$ and $Y$ be random variables having common support $[0, \infty)$, distribution functions $F$ and $G$, and Lebesgue density functions $f$ and $g$, respectively. Let $\bar{F}=1-F$ and $\bar{G}=1-G$ be the survival functions. Also, assume that $f$ and $g$ take positive values on $[0, \infty)$.

Received 16 February 2010; revision received 29 November 2010.

* Postal address: Department of Mathematics and Statistics, Indian Institute of Technology Kanpur, Kanpur, India.

** Email address: neeraj@iitk.ac.in

*** Email address: amishra@iitk.ac.in

**** Email address: idd@iitk.ac.in 
Definition 1.1. ([11].) The random variable $X$ is said to be smaller than $Y$ in the

(i) likelihood ratio order (written as $X \leq \operatorname{lr} Y$ ) if $g(t) / f(t)$ is increasing in $t \in[0, \infty)$;

(ii) usual stochastic order (written as $X \leq_{\text {st }} Y$ ) if $\bar{F}(t) \leq \bar{G}(t)$ for all $t \in[0, \infty)$;

(iii) hazard rate order (written as $X \leq \mathrm{hr} Y$ ) if $\bar{G}(t) / \bar{F}(t)$ is increasing in $t \in[0, \infty)$;

(iv) reversed hazard rate order (written as $X \leq_{\mathrm{rh}} Y$ ) if $G(t) / F(t)$ is increasing in $t \in(0, \infty)$;

(v) increasing convex order (written as $\left.X \leq_{\mathrm{icx}} Y\right)$ if $\int_{x}^{\infty} \mathrm{P}(Y>t) \mathrm{d} t \geq \int_{x}^{\infty} \mathrm{P}(X>t) \mathrm{d} t$ for all $x \in[0, \infty)$;

(vi) increasing concave order (written as $\left.X \leq_{\text {icv }} Y\right)$ if $\int_{0}^{x} \mathrm{P}(Y>t) \mathrm{d} t \geq \int_{0}^{x} \mathrm{P}(X>t) \mathrm{d} t$ for all $x \in[0, \infty)$;

(vii) stochastic precedence order (written as $X \leq_{\text {sp }} Y$ ) if $\mathrm{P}(X>Y) \leq \mathrm{P}(X<Y)$.

Definition 1.2. ([11].) The random variable $X$ is said to have an increasing failure rate (IFR) if $\bar{F}(\cdot)$ is log-concave on $[0, \infty)$.

For equivalent definitions and properties of various stochastic orders and ageing classes, we refer the reader to [4], [8], and [11].

Consider a series (or parallel) system consisting of components $C_{1}, C_{2}, \ldots, C_{n}$ having random lifetimes $X_{1}, X_{2}, \ldots, X_{n}$, respectively. Suppose that we also have spares $R, R_{1}$, and $R_{2}$ having random lifetimes $X, Y_{1}$, and $Y_{2}$, respectively. Assume that nonnegative random variables $X_{1}, X_{2}, \ldots, X_{n}, X, Y_{1}$, and $Y_{2}$ are statistically independent. Boland et al. [2], Singh and Misra [12], and Li and $\mathrm{Hu}$ [5] considered a model in which a spare $R$ is available for standby redundancy. The available spare can be allocated either to component $C_{1}$ or to component $C_{2}$. We can decide which of these two allocations is better (with respect to some performance characteristics of the resulting systems) by making stochastic comparisons on

$$
V_{1}=\wedge\left\{X_{1}+X, X_{2}, X_{3}, \ldots, X_{n}\right\} \quad \text { and } \quad V_{2}=\wedge\left\{X_{1}, X_{2}+X, X_{3}, \ldots, X_{n}\right\}
$$

for the series system, and on

$$
V_{1}^{\prime}=\vee\left\{X_{1}+X, X_{2}, X_{3}, \ldots, X_{n}\right\} \quad \text { and } \quad V_{2}^{\prime}=\vee\left\{X_{1}, X_{2}+X, X_{3}, \ldots, X_{n}\right\}
$$

for the parallel system; here the symbols ' $\wedge$ ' and ' $\vee$ ' represent $\min$ and max, respectively. Boland et al. [2] proved that if $X_{1} \leq_{\mathrm{hr}} X_{2}$ then $V_{2} \leq_{\mathrm{st}} V_{1}$ and if $X_{1} \leq_{\mathrm{rh}} X_{2}$ then $V_{1}^{\prime} \leq_{\mathrm{st}} V_{2}^{\prime}$. Singh and Misra [12] established that if $X_{1} \leq_{\mathrm{st}} X_{2}$ then $V_{2} \leq_{\mathrm{sp}} V_{1}$ and $V_{1}^{\prime} \leq_{\mathrm{sp}} V_{2}^{\prime}$. Li and Hu [5] proved that if $X_{1} \leq_{\text {icv }} X_{2}$ and $X_{1}, X_{3}, \ldots, X_{n}$ have convex survival functions, then $V_{2} \leq_{\text {sp }} V_{1}$. They also proved that if $X_{1}$ or $X_{2}$ has a convex survival function and if $X_{1} \leq_{\text {icv }} X_{2}$, then $\vee\left\{X_{1}+X, X_{2}\right\} \leq_{\text {sp }} \vee\left\{X_{1}, X_{2}+X\right\}$.

Following the ideas of Valdés and Zequeira [13] and Romera et al. [9], we consider two models which are mathematically more general than the model considered in [2], [5], and [12]. In the first model we have two spares $R_{1}$ and $R_{2}$ (possibly identical), and, due to some constraints, we can use only one of them: either $R_{1}$ with $C_{1}$, or $R_{2}$ with $C_{2}$. To decide which of these two allocations is better, we make stochastic comparisons on

$$
U_{1}=\wedge\left\{X_{1}+Y_{1}, X_{2}, X_{3}, \ldots, X_{n}\right\} \quad \text { and } \quad U_{2}=\wedge\left\{X_{1}, X_{2}+Y_{2}, X_{3}, \ldots, X_{n}\right\}
$$

and on

$$
U_{1}^{\prime}=\vee\left\{X_{1}+Y_{1}, X_{2}, X_{3}, \ldots, X_{n}\right\} \quad \text { and } \quad U_{2}^{\prime}=\vee\left\{X_{1}, X_{2}+Y_{2}, X_{3}, \ldots, X_{n}\right\} .
$$


Note that if $Y_{1}$ and $Y_{2}$ are identically distributed then this model reduces to the model considered in [2], [5], and [12]. In Section 2 we provide conditions under which $U_{2} \leq_{\mathrm{icv}} U_{1}, U_{2} \leq \mathrm{hr} U_{1}$, $U_{2} \leq_{\text {sp }} U_{1}, U_{1}^{\prime} \leq_{\text {icx }} U_{2}^{\prime}$, and $U_{1}^{\prime} \leq_{\text {sp }} U_{2}^{\prime}$ hold. For allocation of active redundancy, this model was considered in [7], [9], and [13]. A practical situation where the above model may be of interest is described in the following example, which is similar to Example 3.2 discussed in [3].

Example 1.1. Two safety devices $S_{1}$ and $S_{2}$ run on batteries $B_{1}$ and $B_{2}$, respectively. The batteries $B_{1}$ and $B_{2}$ are of different types and have lifetimes $X_{1}$ and $X_{2}$, respectively. Suppose that two new types of battery, $B_{1}^{\prime}$ and $B_{2}^{\prime}$, are available in the market and that $B_{i}^{\prime}$ is compatible with only $S_{i}, i=1,2$. Let $Y_{i}$ denote the lifetime of $B_{i}^{\prime}, i=1,2$. Owing to a limited budget, let us assume that we can afford a spare battery for one of the devices only. If our aim is to optimize the time during which both devices will function, stochastic comparisons on $U_{1}=\wedge\left\{X_{1}+Y_{1}, X_{2}\right\}$ and $U_{2}=\wedge\left\{X_{1}, X_{2}+Y_{2}\right\}$ may be of interest.

In the second model we have two spares $R_{1}$ and $R_{2}$ which can be used in one of the following two ways: $R_{1}$ with $C_{2}$ and $R_{2}$ with $C_{1}$, or $R_{1}$ with $C_{1}$ and $R_{2}$ with $C_{2}$. In Section 3 we compare these two methods of allocation of spares through stochastic comparisons on

$$
Z_{1}=\wedge\left\{X_{1}+Y_{2}, X_{2}+Y_{1}, X_{3}, \ldots, X_{n}\right\} \quad \text { and } \quad Z_{2}=\wedge\left\{X_{1}+Y_{1}, X_{2}+Y_{2}, X_{3}, \ldots, X_{n}\right\}
$$

for the series system, and on

$$
Z_{1}^{\prime}=\vee\left\{X_{1}+Y_{2}, X_{2}+Y_{1}, X_{3}, \ldots, X_{n}\right\} \quad \text { and } \quad Z_{2}^{\prime}=\vee\left\{X_{1}+Y_{1}, X_{2}+Y_{2}, X_{3}, \ldots, X_{n}\right\}
$$

for the parallel system. Note that if $\mathrm{P}\left(Y_{2}=0\right)=1$ then this model reduces to the model considered in [2], [5], and [12]. In Section 3 we derive conditions under which $Z_{2} \leq_{\mathrm{sp}} Z_{1}$ and $Z_{1}^{\prime} \leq_{\text {sp }} Z_{2}^{\prime}$ hold. For allocation of active redundancy, this model was considered in [9] and [14]. The following example illustrates a situation where the above model may be of interest.

Example 1.2. In Example 1.1, suppose that each of $B_{1}^{\prime}$ and $B_{2}^{\prime}$ is compatible with both $S_{1}$ and $S_{2}$. Now suppose that we do not have a budgetary constraint and, thus, spare batteries of both types can be used, i.e. we can use either $B_{1}^{\prime}$ with $S_{1}$ and $B_{2}^{\prime}$ with $S_{2}$, or $B_{1}^{\prime}$ with $S_{2}$ and $B_{2}^{\prime}$ with $S_{1}$. For optimizing the time during which both devices will function, we may be interested in stochastic comparisons on $Z_{1}=\wedge\left\{X_{1}+Y_{2}, X_{2}+Y_{1}\right\}$ and $Z_{2}=\wedge\left\{X_{1}+Y_{1}, X_{2}+Y_{2}\right\}$.

Throughout this paper, increasing and decreasing are used to mean nondecreasing and nonincreasing, respectively. Moreover, the common support of all the random variables considered in the paper is assumed to be $[0, \infty)$, which we denote by $\mathbb{R}_{+}$. For random variables $X$ and $Y$, we write $X={ }_{\text {st }} Y$ to indicate that $X$ and $Y$ have the same distribution. For $x, y \in \mathbb{R}:=(-\infty, \infty)$, we define $I(x>y)=1$ if $x>y$ and $I(x>y)=0$ if $x \leq y$. Let $F_{i}, \bar{F}_{i}$, and $f_{i}$ respectively denote the distribution function, the survival function, and the Lebesgue density function of $X_{i}, i=1,2, \ldots, n$. Furthermore, let $G_{i}, \bar{G}_{i}$, and $g_{i}$ respectively denote the distribution function, the survival function, and the Lebesgue density function of $Y_{i}, i=1,2$.

\section{Allocation of one standby redundancy}

In this section we will make stochastic comparisons between $U_{1}$ and $U_{2}$ and also between $U_{1}^{\prime}$ and $U_{2}^{\prime}$. It may be worth mentioning here that any result on the stochastic comparison between $U_{1}$ and $U_{2}$ (or $U_{1}^{\prime}$ and $U_{2}^{\prime}$ ) yields, as a particular case, a result on the stochastic comparison between $V_{1}$ and $V_{2}$ (or $V_{1}^{\prime}$ and $V_{2}^{\prime}$ ). 
Theorem 2.1. If $X_{1} \leq_{\mathrm{st}} X_{2}$ and $Y_{2} \leq_{\mathrm{icv}} Y_{1}$, then $U_{2} \leq_{\mathrm{icv}} U_{1}$.

Proof. Let $\bar{H}(t)=\prod_{i=3}^{n} \bar{F}_{i}(t), t \in \mathbb{R}_{+}$. Then, it suffices to prove that

$$
\begin{aligned}
\Delta_{1}(x) & =\int_{0}^{x} \mathrm{P}\left(U_{1}>t\right) \mathrm{d} t-\int_{0}^{x} \mathrm{P}\left(U_{2}>t\right) \mathrm{d} t \\
& =\int_{0}^{x} \bar{H}(t) \bar{F}_{2}(t) \mathrm{P}\left(X_{1}+Y_{1}>t\right) \mathrm{d} t-\int_{0}^{x} \bar{H}(t) \bar{F}_{1}(t) \mathrm{P}\left(X_{2}+Y_{2}>t\right) \mathrm{d} t
\end{aligned}
$$

is nonnegative for every $x \in \mathbb{R}_{+}$. Since the increasing concave order is closed under convolution (see Theorem 4.A.8(d) of [11]), we have $X_{1}+Y_{2} \leq_{\text {icv }} X_{1}+Y_{1}$, i.e.

$$
\int_{0}^{x} \mathrm{P}\left(X_{1}+Y_{1}>t\right) \mathrm{d} t \geq \int_{0}^{x} \mathrm{P}\left(X_{1}+Y_{2}>t\right) \mathrm{d} t \quad \text { for all } x \in \mathbb{R}_{+} .
$$

Now by using Theorem 7.3 of [1, Chapter 4] we obtain

$$
\int_{0}^{x} \bar{H}(t) \bar{F}_{2}(t) \mathrm{P}\left(X_{1}+Y_{1}>t\right) \mathrm{d} t \geq \int_{0}^{x} \bar{H}(t) \bar{F}_{2}(t) \mathrm{P}\left(X_{1}+Y_{2}>t\right) \mathrm{d} t \quad \text { for all } x \in \mathbb{R}_{+} .
$$

Therefore, for every $x \in \mathbb{R}_{+}$,

$$
\Delta_{1}(x) \geq \int_{0}^{x} \bar{H}(t) \bar{F}_{2}(t) \mathrm{P}\left(X_{1}+Y_{2}>t\right) \mathrm{d} t-\int_{0}^{x} \bar{H}(t) \bar{F}_{1}(t) \mathrm{P}\left(X_{2}+Y_{2}>t\right) \mathrm{d} t .
$$

Moreover, for all $x \in \mathbb{R}_{+}$,

$$
\begin{aligned}
\int_{0}^{x} \bar{H}(t) & \bar{F}_{2}(t) \mathrm{P}\left(X_{1}+Y_{2}>t\right) \mathrm{d} t-\int_{0}^{x} \bar{H}(t) \bar{F}_{1}(t) \mathrm{P}\left(X_{2}+Y_{2}>t\right) \mathrm{d} t \\
= & \int_{0}^{x} \int_{t}^{\infty} \bar{H}(t) \mathrm{P}\left(X_{1}+Y_{2}>t\right) f_{2}(u) \mathrm{d} u \mathrm{~d} t \\
& -\int_{0}^{x} \bar{H}(t) \bar{F}_{1}(t)\left(\int_{0}^{t} \bar{G}_{2}(t-u) f_{2}(u) \mathrm{d} u+\int_{t}^{\infty} f_{2}(u) \mathrm{d} u\right) \mathrm{d} t \\
= & \int_{0}^{\infty}\left(\int_{0}^{u \wedge x} \bar{H}(t)\left[\mathrm{P}\left(X_{1}+Y_{2}>t\right)-\bar{F}_{1}(t)\right] \mathrm{d} t\right) f_{2}(u) \mathrm{d} u \\
& -\int_{0}^{x}\left(\int_{u}^{x} \bar{H}(t) \bar{F}_{1}(t) \bar{G}_{2}(t-u) \mathrm{d} t\right) f_{2}(u) \mathrm{d} u \quad \text { (using Fubini's theorem) } \\
= & \mathrm{E}\left[\psi_{1}\left(X_{2}\right)\right],
\end{aligned}
$$

where

$$
\psi_{1}(y)=\left\{\begin{array}{cl}
\int_{0}^{y} \bar{H}(t)\left[\mathrm{P}\left(X_{1}+Y_{2}>t\right)-\bar{F}_{1}(t)\right] \mathrm{d} t & \\
-\int_{0}^{x-y} \bar{H}(t+y) \bar{F}_{1}(t+y) \bar{G}_{2}(t) \mathrm{d} t & \text { if } 0 \leq y \leq x, \\
\int_{0}^{x} \bar{H}(t)\left[\mathrm{P}\left(X_{1}+Y_{2}>t\right)-\bar{F}_{1}(t)\right] \mathrm{d} t & \text { if } y>x .
\end{array}\right.
$$

Clearly, for every fixed $x \in \mathbb{R}_{+}, \psi_{1}(y)$ is an increasing function of $y$ on $\mathbb{R}_{+}$. Let $\hat{X}_{1}={ }_{\text {st }} X_{1}$ be such that $\hat{X}_{1}, X_{1}, X_{2}, \ldots, X_{n}, Y_{1}$, and $Y_{2}$ are independent random variables. Then $\hat{X}_{1} \leq_{\text {st }} X_{2}$, 
and, thus, for every $x \in \mathbb{R}_{+}$,

$$
\begin{aligned}
\Delta_{1}(x) & \geq \mathrm{E}\left[\psi_{1}\left(X_{2}\right)\right] \\
& \geq \mathrm{E}\left[\psi_{1}\left(\hat{X}_{1}\right)\right] \\
& =\int_{0}^{x} \bar{H}(t) \mathrm{P}\left(\hat{X}_{1}>t\right) \mathrm{P}\left(X_{1}+Y_{2}>t\right) \mathrm{d} t-\int_{0}^{x} \bar{H}(t) \bar{F}_{1}(t) \mathrm{P}\left(\hat{X}_{1}+Y_{2}>t\right) \mathrm{d} t \\
& =0 .
\end{aligned}
$$

Next we will compare $U_{1}^{\prime}$ and $U_{2}^{\prime}$ with respect to the increasing convex order.

Theorem 2.2. If $X_{1} \leq_{\mathrm{st}} X_{2}$ and $Y_{1} \leq_{\mathrm{icx}} Y_{2}$, then $U_{1}^{\prime} \leq_{\mathrm{icx}} U_{2}^{\prime}$.

Proof. Let $K(t)=\prod_{i=3}^{n} F_{i}(t), t \in \mathbb{R}_{+}$. Then, it suffices to prove that

$$
\begin{aligned}
\Delta_{2}(x) & =\int_{x}^{\infty}\left[\mathrm{P}\left(U_{2}^{\prime}>t\right)-\mathrm{P}\left(U_{1}^{\prime}>t\right)\right] \mathrm{d} t \\
& =\int_{x}^{\infty} K(t)\left[F_{2}(t) \mathrm{P}\left(X_{1}+Y_{1} \leq t\right)-F_{1}(t) \mathrm{P}\left(X_{2}+Y_{2} \leq t\right)\right] \mathrm{d} t
\end{aligned}
$$

is nonnegative for every $x \in \mathbb{R}_{+}$. Preservation of the increasing convex order under convolution (see Theorem 4.A.8(d) of [11]) implies that $X_{2}+Y_{1} \leq_{\text {icx }} X_{2}+Y_{2}$, i.e.

$$
\int_{x}^{\infty} \mathrm{P}\left(X_{2}+Y_{2}>t\right) \mathrm{d} t \geq \int_{x}^{\infty} \mathrm{P}\left(X_{2}+Y_{1}>t\right) \mathrm{d} t \quad \text { for all } x \in \mathbb{R}_{+} .
$$

Now Theorem 7.4 of [1, Chapter 4] yields, for every $x \in \mathbb{R}_{+}$,

$$
\int_{x}^{\infty}\left[1-K(t) F_{1}(t) \mathrm{P}\left(X_{2}+Y_{2} \leq t\right)\right] \mathrm{d} t \geq \int_{x}^{\infty}\left[1-K(t) F_{1}(t) \mathrm{P}\left(X_{2}+Y_{1} \leq t\right)\right] \mathrm{d} t,
$$

i.e.

$$
\Delta_{2}(x) \geq \int_{x}^{\infty} K(t)\left[F_{2}(t) \mathrm{P}\left(X_{1}+Y_{1} \leq t\right)-F_{1}(t) \mathrm{P}\left(X_{2}+Y_{1} \leq t\right)\right] \mathrm{d} t \quad \text { for all } x \in \mathbb{R}_{+} .
$$

Moreover, for every $x \in \mathbb{R}_{+}$,

$$
\begin{aligned}
\int_{x}^{\infty} & K(t)\left[F_{2}(t) \mathrm{P}\left(X_{1}+Y_{1} \leq t\right)-F_{1}(t) \mathrm{P}\left(X_{2}+Y_{1} \leq t\right)\right] \mathrm{d} t \\
& =\int_{x}^{\infty} \int_{0}^{t} K(t)\left[\mathrm{P}\left(X_{1}+Y_{1} \leq t\right)-F_{1}(t) G_{1}(t-u)\right] f_{2}(u) \mathrm{d} u \mathrm{~d} t \\
& =\int_{0}^{\infty}\left(\int_{u \vee x}^{\infty} K(t)\left[\mathrm{P}\left(X_{1}+Y_{1} \leq t\right)-F_{1}(t) G_{1}(t-u)\right] \mathrm{d} t\right) f_{2}(u) \mathrm{d} u \\
& =\mathrm{E}\left[\psi_{2}\left(X_{2}\right)\right]
\end{aligned}
$$

where

$$
\psi_{2}(y)=\int_{y \vee x}^{\infty} K(t)\left[\mathrm{P}\left(X_{1}+Y_{1} \leq t\right)-F_{1}(t) G_{1}(t-y)\right] \mathrm{d} t, \quad y \in \mathbb{R}_{+} .
$$


Clearly, $\psi_{2}(y)$ is increasing in $y \in[0, x]$. Furthermore, for $y>x$,

$$
\begin{aligned}
\frac{\mathrm{d}}{\mathrm{d} y} \psi_{2}(y) & =\int_{y}^{\infty} K(t) F_{1}(t) g_{1}(t-y) \mathrm{d} t-K(y) \mathrm{P}\left(X_{1}+Y_{1} \leq y\right) \\
& \geq K(y)\left[F_{1}(y)-\mathrm{P}\left(X_{1}+Y_{1} \leq y\right)\right] \\
& \geq 0 .
\end{aligned}
$$

Thus, $\psi_{2}(y)$ is increasing in $y \in \mathbb{R}_{+}$. Let $\hat{X}_{1}={ }_{\text {st }} X_{1}$ be such that $\hat{X}_{1}, X_{1}, X_{2}, \ldots, X_{n}, Y_{1}$, and $Y_{2}$ are independent random variables. Then $\hat{X}_{1} \leq_{\text {st }} X_{2}$ and, thus, for every $x \in \mathbb{R}_{+}$,

$$
\begin{aligned}
\Delta_{2}(x) & \geq \mathrm{E}\left[\psi_{2}\left(X_{2}\right)\right] \\
& \geq \mathrm{E}\left[\psi_{2}\left(\hat{X}_{1}\right)\right] \\
& =\int_{x}^{\infty} K(t)\left[\mathrm{P}\left(\hat{X}_{1} \leq t\right) \mathrm{P}\left(X_{1}+Y_{1} \leq t\right)-F_{1}(t) \mathrm{P}\left(\hat{X}_{1}+Y_{1} \leq t\right)\right] \mathrm{d} t \\
& =0 .
\end{aligned}
$$

The following lemma, which may be of independent interest, will be useful in the stochastic comparison of $U_{1}$ and $U_{2}$ with respect to the hazard rate ordering.

Lemma 2.1. Let $\mathcal{X}, \mathcal{Y} \subseteq \mathbb{R}$, and let $g: \mathcal{X} \times \mathcal{y} \rightarrow \mathbb{R}_{+}$and $\psi: \mathcal{X} \times \mathcal{Y} \rightarrow \mathbb{R}$ be such that

(i) $g(x, y)$ is $T P_{2}$ on $X \times y$ and, for each $y \in \mathcal{Y}, \int_{X} g(x, y) \mathrm{d} x>0$;

(ii) for each $y \in \mathcal{Y}, \psi(x, y)$ is increasing in $x \in \mathcal{X}$;

(iii) for each $x \in \mathcal{X}, \psi(x, y)$ is increasing in $y \in \mathcal{Y}$.

Then the function

$$
K(y)=\frac{\int_{\mathcal{X}} \psi(x, y) g(x, y) \mathrm{d} x}{\int_{X} g(x, y) \mathrm{d} x}
$$

is increasing in $y \in y$. If $\psi(x, y)$ is decreasing in (ii) and (iii), then the function $K(y)$ is decreasing in $y \in \mathcal{Y}$.

Proof. In view of (i), (ii), and (iii) we have, for $y_{1} \leq y_{2}$,

$$
\begin{aligned}
{\left[K\left(y_{2}\right)\right.} & \left.-K\left(y_{1}\right)\right] \int_{X} \int_{X} g\left(x_{1}, y_{1}\right) g\left(x_{2}, y_{2}\right) \mathrm{d} x_{2} \mathrm{~d} x_{1} \\
= & \int_{X} \int_{X} \psi\left(x_{2}, y_{2}\right) g\left(x_{2}, y_{2}\right) g\left(x_{1}, y_{1}\right) \mathrm{d} x_{2} \mathrm{~d} x_{1} \\
& -\int_{X} \int_{X} \psi\left(x_{2}, y_{1}\right) g\left(x_{2}, y_{1}\right) g\left(x_{1}, y_{2}\right) \mathrm{d} x_{2} \mathrm{~d} x_{1} \\
\geq & \int_{X} \int_{X} \psi\left(x_{2}, y_{1}\right)\left[g\left(x_{2}, y_{2}\right) g\left(x_{1}, y_{1}\right)-g\left(x_{2}, y_{1}\right) g\left(x_{1}, y_{2}\right)\right] \mathrm{d} x_{2} \mathrm{~d} x_{1} \\
= & \int_{X} \int_{X}\left[\psi\left(x_{2}, y_{1}\right)-\psi\left(x_{1}, y_{1}\right)\right]\left[g\left(x_{2}, y_{2}\right) g\left(x_{1}, y_{1}\right)-g\left(x_{2}, y_{1}\right) g\left(x_{1}, y_{2}\right)\right] \\
\geq & 0, \quad \times I\left(x_{2}>x_{1}\right) \mathrm{d} x_{2} \mathrm{~d} x_{1}
\end{aligned}
$$

i.e. $K\left(y_{2}\right) \geq K\left(y_{1}\right)$. If $\psi(x, y)$ is decreasing in (ii) and (iii), then all the ' $\geq$ ' above are replaced by ' $\leq$ '. 
Theorem 2.3. Suppose that $X_{1}$ or $X_{2}$ has IFR, $\bar{F}_{2}(t) / \bar{F}_{1}(t)$ is log-convex in $t \in \mathbb{R}_{+}, X_{1} \leq \mathrm{hr}$ $X_{2}$, and that $Y_{2} \leq_{\mathrm{lr}} Y_{1}$. Then $U_{2} \leq_{\mathrm{hr}} U_{1}$.

Proof. First suppose that $X_{2}$ has IFR. For $t \in \mathbb{R}_{+}$, we have

$$
\frac{\mathrm{P}\left(U_{1}>t\right)}{\mathrm{P}\left(U_{2}>t\right)}=\frac{\mathrm{P}\left(X_{1}+Y_{1}>t\right) \bar{F}_{2}(t)}{\mathrm{P}\left(X_{2}+Y_{2}>t\right) \bar{F}_{1}(t)}=\frac{\int_{0}^{\infty} \psi(u, t) g(u, t) \mathrm{d} u}{\int_{0}^{\infty} g(u, t) \mathrm{d} u}=h_{1}(t), \quad \text { say, }
$$

where, for $u, t \in \mathbb{R}_{+}$,

$$
\psi(u, t)=\frac{\bar{F}_{1}(t-u) \bar{F}_{2}(t) g_{1}(u)}{\bar{F}_{2}(t-u) \bar{F}_{1}(t) g_{2}(u)} \quad \text { and } \quad g(u, t)=\frac{\bar{F}_{2}(t-u)}{\bar{F}_{2}(t)} g_{2}(u) .
$$

The IFR property of $X_{2}$ implies that $\bar{F}_{2}(t-u) / \bar{F}_{2}(t)$, and, hence, $g(u, t)$, is $\mathrm{TP}_{2}$. Now we will show that, for each fixed $t \in \mathbb{R}_{+}$or $u \in \mathbb{R}_{+}, \psi(u, t)$ is increasing in $u \in \mathbb{R}_{+}$or, respectively, $t \in \mathbb{R}_{+}$. The assumptions that $X_{1} \leq \mathrm{hr} X_{2}$ and $Y_{2} \leq \operatorname{lr} Y_{1}$ respectively imply that $\bar{F}_{1}(t-u) / \bar{F}_{2}(t-u)$ and $g_{1}(u) / g_{2}(u)$ are increasing in $u \in \mathbb{R}_{+}$for each fixed $t \in \mathbb{R}_{+}$. Therefore, for each fixed $t \in \mathbb{R}_{+}, \psi(u, t)$ is increasing in $u \in \mathbb{R}_{+}$. For fixed $u \in \mathbb{R}_{+}$, we have

$$
\psi(u, t)= \begin{cases}\frac{\bar{F}_{2}(t) g_{1}(u)}{\bar{F}_{1}(t) g_{2}(u)} & \text { if } 0 \leq t<u, \\ \bar{F}_{2}(t) \bar{F}_{1}(t-u) g_{1}(u) & \text { if } t \geq u . \\ \frac{\bar{F}_{1}(t) \bar{F}_{2}(t-u) g_{2}(u)}{} & \end{cases}
$$

The assumption that $X_{1} \leq \mathrm{hr} X_{2}$ implies that $\psi(u, t)$ is increasing in $t \in[0, u)$. Moreover, for $t \geq u$, we have

$$
\ln \psi(u, t)=\ln \frac{\bar{F}_{2}(t)}{\bar{F}_{1}(t)}-\ln \frac{\bar{F}_{2}(t-u)}{\bar{F}_{1}(t-u)}+\ln \frac{g_{1}(u)}{g_{2}(u)} .
$$

Since $\bar{F}_{2}(t) / \bar{F}_{1}(t)$ is log-convex in $t \in \mathbb{R}_{+}$, it follows that $\ln \psi(u, t)$ (and so $\psi(u, t)$ ) is increasing in $t \in[u, \infty)$. Now, on using Lemma 2.1, it follows that $h_{1}(t)$ is increasing in $t \in \mathbb{R}_{+}$.

Now consider the case when $X_{1}$ has IFR. We can write

$$
\frac{1}{h_{1}(t)}=\int_{0}^{\infty}\left[\frac{\bar{F}_{2}(t-u) \bar{F}_{1}(t) g_{2}(u)}{\bar{F}_{1}(t-u) \bar{F}_{2}(t) g_{1}(u)}\right] \frac{\bar{F}_{1}(t-u)}{\bar{F}_{1}(t)} g_{1}(u) \mathrm{d} u / \int_{0}^{\infty} \frac{\bar{F}_{1}(t-u)}{\bar{F}_{1}(t)} g_{1}(u) \mathrm{d} u
$$

for $t \in \mathbb{R}_{+}$. Proceeding as in the previous case, we can show that $1 / h_{1}(t)$ is decreasing in $t \in \mathbb{R}_{+}$. This implies that $h_{1}(t)$ is increasing in $t \in \mathbb{R}_{+}$.

The following theorem deals with the stochastic comparison of $U_{1}$ and $U_{2}$ with respect to the stochastic precedence order.

Theorem 2.4. Suppose that $X_{1} \leq \mathrm{icv} X_{2}$, and that $X_{1}, X_{3}, X_{4}, \ldots, X_{n}\left(\right.$ or $\left.X_{2}, X_{3}, X_{4}, \ldots, X_{n}\right)$ have convex survival functions on $\mathbb{R}_{+}$. Then $U_{2} \leq_{\mathrm{sp}} U_{1}$.

Proof. Let $T=\wedge\left\{X_{3}, X_{4}, \ldots, X_{n}\right\}$. Then it is easy to verify that

$$
\left[U_{1}>U_{2}\right]=\left[X_{2}>\wedge\left\{X_{1}, T\right\}\right] \cap\left[T>\wedge\left\{X_{1}, X_{2}+Y_{2}\right\}\right]=\left[X_{2}>X_{1}\right] \cap\left[T>X_{1}\right] .
$$


Let $\bar{H}$ be the survival function of $T$. By symmetry we have

$$
\begin{aligned}
\Delta_{4} & \equiv \mathrm{P}\left(U_{1}>U_{2}\right)-\mathrm{P}\left(U_{2}>U_{1}\right) \\
& =\mathrm{P}\left(X_{2}>X_{1}, T>X_{1}\right)-\mathrm{P}\left(X_{1}>X_{2}, T>X_{2}\right) \\
& =\mathrm{E}\left[\bar{H}\left(X_{1}\right) I\left(X_{2}>X_{1}\right)-\bar{H}\left(X_{2}\right) I\left(X_{1}>X_{2}\right)\right] .
\end{aligned}
$$

Case I: $X_{1}, X_{3}, X_{4}, \ldots, X_{n}$ have convex survival functions. Let $\hat{X}_{1}$ be an independent copy of $X_{1}$. Then, using (2.1), we can write $\Delta_{4}=\mathrm{E}\left[\psi_{3}\left(X_{2}\right)\right]$, where

$$
\psi_{3}(t)=\mathrm{E}\left[\bar{H}\left(\hat{X}_{1}\right) I\left(t>\hat{X}_{1}\right)-\bar{H}(t) I\left(\hat{X}_{1}>t\right)\right], \quad t \in \mathbb{R}_{+} .
$$

Clearly, $\hat{X}_{1} \leq_{\text {icv }} X_{2}$ and $\mathrm{E}\left[\psi_{3}\left(\hat{X}_{1}\right)\right]=0$. Using (2.2), we have

$$
\psi_{3}(t)=\int_{0}^{t} \bar{H}(s) f_{1}(s) \mathrm{d} s-\bar{H}(t) \bar{F}_{1}(t), \quad t \in \mathbb{R}_{+},
$$

and, therefore,

$$
\psi_{3}^{\prime}(t)=\frac{\mathrm{d}}{\mathrm{d} t} \psi_{3}(t)=2 \bar{H}(t) f_{1}(t)+\bar{F}_{1}(t) \sum_{i=3}^{n} f_{i}(t) \prod_{\substack{j=3 \\ j \neq i}}^{n} \bar{F}_{j}(t) \geq 0 \quad \text { for all } t \in \mathbb{R}_{+}
$$

Since $X_{1}, X_{3}, X_{4}, \ldots, X_{n}$ have convex survival functions on $\mathbb{R}_{+}\left(\right.$i.e. $f_{i}(\cdot), i=1,3,4, \ldots, n$ are decreasing on $\mathbb{R}_{+}$), it follows that $\psi_{3}^{\prime}(\cdot)$ is a decreasing function on $\mathbb{R}_{+}$. Thus, $\psi_{3}(\cdot)$ is an increasing concave function on $\mathbb{R}_{+}$. Now, on using $\hat{X}_{1} \leq_{\text {icv }} X_{2}$ we obtain

$$
\Delta_{4}=\mathrm{E}\left[\psi_{3}\left(X_{2}\right)\right] \geq \mathrm{E}\left[\psi_{3}\left(\hat{X}_{1}\right)\right]=0
$$

i.e. $U_{2} \leq_{\mathrm{sp}} U_{1}$.

Case II: $X_{2}, X_{3}, X_{4}, \ldots, X_{n}$ have convex survival functions. Let $\hat{X}_{2}$ be an independent copy of $X_{2}$. Then, using (2.1), we can write $\Delta_{4}=-\mathrm{E}\left[\psi_{4}\left(X_{1}\right)\right]$, where

$$
\psi_{4}(t)=\mathrm{E}\left[\bar{H}\left(\hat{X}_{2}\right) I\left(t>\hat{X}_{2}\right)-\bar{H}(t) I\left(\hat{X}_{2}>t\right)\right], \quad t \in \mathbb{R}_{+} .
$$

Then, $X_{1} \leq_{\text {icv }} \hat{X}_{2}$ and $\mathrm{E}\left[\psi_{4}\left(\hat{X}_{2}\right)\right]=0$. Proceeding as in case I, we can show that $\psi_{4}(\cdot)$ is an increasing concave function on $\mathbb{R}_{+}$. Therefore,

$$
\Delta_{4}=-\mathrm{E}\left[\psi_{4}\left(X_{1}\right)\right] \geq-\mathrm{E}\left[\psi_{4}\left(\hat{X}_{2}\right)\right]=0,
$$

i.e. $U_{2} \leq_{\mathrm{sp}} U_{1}$.

Theorem 2.5. Suppose that $X_{1} \leq_{\mathrm{icx}} X_{2}, Y_{1} \leq_{\mathrm{icx}} Y_{2}$, and that $X_{1}, X_{3}, X_{4}, \ldots, X_{n}$ (or $X_{2}, X_{3}$, $X_{4}, \ldots, X_{n}$ ) have concave survival functions on $\mathbb{R}_{+}$. Then $U_{1}^{\prime} \leq_{\mathrm{sp}} U_{2}^{\prime}$.

Proof. Let $T_{1}=\vee\left\{X_{3}, X_{4}, \ldots, X_{n}\right\}$. Then it can be verified that

$$
\left[U_{2}^{\prime}>U_{1}^{\prime}\right]=\left[W_{2}>W_{1}\right] \cap\left[W_{2}>T_{1}\right]
$$

where $W_{i}=X_{i}+Y_{i}, i=1,2$. 
Let $K$ be the distribution function of $T_{1}$, and let $\pi_{i}(\cdot)$ and $\Pi_{i}(\cdot)$ respectively denote the density function and the distribution function of $W_{i}, i=1,2$. Using symmetry, we have

$$
\begin{aligned}
\Delta_{5} & \equiv \mathrm{P}\left(U_{2}^{\prime}>U_{1}^{\prime}\right)-\mathrm{P}\left(U_{1}^{\prime}>U_{2}^{\prime}\right) \\
& =\mathrm{P}\left(W_{2}>W_{1}, W_{2}>T_{1}\right)-\mathrm{P}\left(W_{1}>W_{2}, W_{1}>T_{1}\right) \\
& =\mathrm{E}\left[K\left(W_{2}\right) I\left(W_{2}>W_{1}\right)-K\left(W_{1}\right) I\left(W_{1}>W_{2}\right)\right] .
\end{aligned}
$$

Case I: $X_{1}, X_{3}, X_{4}, \ldots, X_{n}$ have concave survival functions. Let $\hat{W}_{1}$ be an independent copy of $W_{1}$. Then (2.3) can be written as $\Delta_{5}=\mathrm{E}\left[\psi_{5}\left(W_{2}\right)\right]$, where

$$
\begin{aligned}
\psi_{5}(t) & =\mathrm{E}\left[K(t) I\left(t>\hat{W}_{1}\right)-K\left(\hat{W}_{1}\right) I\left(\hat{W}_{1}>t\right)\right] \\
& =K(t) \Pi_{1}(t)-\int_{t}^{\infty} K(s) \pi_{1}(s) \mathrm{d} s, \quad t \in \mathbb{R}_{+} .
\end{aligned}
$$

Evidently, $\mathrm{E}\left[\psi_{5}\left(\hat{W}_{1}\right)\right]=0$ and from $(2.4)$ we obtain

$$
\psi_{5}^{\prime}(t)=\frac{\mathrm{d}}{\mathrm{d} t} \psi_{5}(t)=2 K(t) \pi_{1}(t)+\Pi_{1}(t) \sum_{i=3}^{n} f_{i}(t) \prod_{\substack{j=3 \\ j \neq i}}^{n} F_{j}(t) \geq 0 \quad \text { for all } t \in \mathbb{R}_{+} .
$$

Using the concavity of the survival function of $X_{1}$, it can be easily verified that $\pi_{1}(\cdot)$ is increasing on $\mathbb{R}_{+}$. Also, the concavity of the survival function of $X_{i}$ implies that $f_{i}(\cdot)$ is increasing on $\mathbb{R}_{+}, i=3,4, \ldots, n$. Therefore, it follows that $\psi_{5}(\cdot)$ is an increasing convex function on $\mathbb{R}_{+}$. Since the increasing convex order is closed under convolution (see Theorem 4.A.8(d) of [11]), we have $W_{1} \leq_{\text {icx }} W_{2}\left(\right.$ or $\left.\hat{W}_{1} \leq_{\text {icx }} W_{2}\right)$. Consequently,

$$
\Delta_{5}=\mathrm{E}\left[\psi_{5}\left(W_{2}\right)\right] \geq \mathrm{E}\left[\psi_{5}\left(\hat{W}_{1}\right)\right]=0,
$$

i.e. $U_{1}^{\prime} \leq_{\mathrm{sp}} U_{2}^{\prime}$.

Case II: $X_{2}, X_{3}, X_{4}, \ldots, X_{n}$ have concave survival functions. Let $\hat{W}_{2}$ be an independent copy of $W_{2}$ so that we have $\Delta_{5}=-\mathrm{E}\left[\psi_{6}\left(W_{1}\right)\right]$, where

$$
\psi_{6}(t)=\mathrm{E}\left[K(t) I\left(t>\hat{W}_{2}\right)-K\left(\hat{W}_{2}\right) I\left(\hat{W}_{2}>t\right)\right], \quad t \in \mathbb{R}_{+} .
$$

Then $\mathrm{E}\left[\psi_{6}\left(\hat{W}_{2}\right)\right]=0$. Proceeding as in case I, we can show that

$$
\Delta_{5}=-\mathrm{E}\left[\psi_{6}\left(W_{1}\right)\right] \geq-\mathrm{E}\left[\psi_{6}\left(\hat{W}_{2}\right)\right]=0,
$$

i.e. $U_{1}^{\prime} \leq \mathrm{sp} U_{2}^{\prime}$.

Corollary 2.1 below follows from the above theorems on taking $Y_{i}=_{\text {st }} X, i=1,2$.

Corollary 2.1. (i) If $X_{1} \leq_{\mathrm{st}} X_{2}$ then $V_{2} \leq_{\mathrm{icv}} V_{1}$ and $V_{1}^{\prime} \leq_{\mathrm{icx}} V_{2}^{\prime}$.

(ii) Suppose that $X_{1}$ or $X_{2}$ has IFR, $\bar{F}_{2}(t) / \bar{F}_{1}(t)$ is log-convex in $t \in \mathbb{R}_{+}$, and that $X_{1} \leq \mathrm{hr} X_{2}$. Then $V_{2} \leq_{\mathrm{hr}} V_{1}$.

(iii) Suppose that $X_{1} \leq_{\mathrm{icv}} X_{2}$ and that $X_{1}, X_{3}, X_{4}, \ldots, X_{n}$ have convex survival functions on $\mathbb{R}_{+}$. Then $V_{2} \leq_{\text {sp }} V_{1}$.

(iv) Suppose that $X_{1} \leq_{\mathrm{icx}} X_{2}$ and that $X_{2}, X_{3}, X_{4}, \ldots, X_{n}$ have concave survival functions on $\mathbb{R}_{+}$. Then $V_{1}^{\prime} \leq_{\text {sp }} V_{2}^{\prime}$. 
Remark 2.1. (i) On recursively using Theorem 2.1 and Corollary 2.1(i), we have the following assertions.

(a) If $X_{1} \leq_{\mathrm{st}} X_{2} \leq_{\mathrm{st}} \cdots \leq_{\mathrm{st}} X_{n}$ and $Y_{n} \leq_{\mathrm{icv}} Y_{n-1} \leq_{\mathrm{icv}} \cdots \leq_{\mathrm{icv}} Y_{1}$, then $U_{n} \leq_{\mathrm{icv}} U_{n-1} \leq_{\mathrm{icv}}$ $\cdots \leq_{\text {icv }} U_{1}$, where $U_{i}=\wedge\left\{X_{1}, \ldots, X_{i-1}, X_{i}+Y_{i}, X_{i+1}, \ldots, X_{n}\right\}, i=1,2, \ldots, n$.

(b) If $X_{1} \leq_{\mathrm{st}} X_{2} \leq_{\mathrm{st}} \cdots \leq_{\mathrm{st}} X_{n}$ then $V_{n} \leq_{\mathrm{icv}} V_{n-1} \leq_{\mathrm{icv}} \cdots \leq_{\mathrm{icv}} V_{1}$, where $V_{i}=$ $\wedge\left\{X_{1}, \ldots, X_{i-1}, X_{i}+X, X_{i+1}, \ldots, X_{n}\right\}, i=1,2, \ldots, n$.

(ii) On using Theorem 2.2 and Corollary 2.1(i), we have the following assertions.

(a) If $X_{1} \leq_{\mathrm{st}} X_{2} \leq_{\mathrm{st}} \cdots \leq_{\mathrm{st}} X_{n}$ and $Y_{1} \leq_{\mathrm{icx}} Y_{2} \leq_{\mathrm{icx}} \cdots \leq_{\mathrm{icx}} Y_{n}$, then $U_{1}^{\prime} \leq_{\mathrm{icx}} U_{2}^{\prime} \leq_{\mathrm{icx}}$ $\cdots \leq_{\text {icx }} U_{n}^{\prime}$, where $U_{i}^{\prime}=\vee\left\{X_{1}, \ldots, X_{i-1}, X_{i}+Y_{i}, X_{i+1}, \ldots, X_{n}\right\}, i=1,2, \ldots, n$.

(b) If $X_{1} \leq_{\text {st }} X_{2} \leq_{\text {st }} \cdots \leq_{\text {st }} X_{n}$ then $V_{1}^{\prime} \leq_{\text {icx }} V_{2}^{\prime} \leq_{\text {icx }} \cdots \leq_{\text {icx }} V_{n}^{\prime}$, where $V_{i}^{\prime}=\vee\left\{X_{1}, \ldots\right.$, $\left.X_{i-1}, X_{i}+X, X_{i+1}, \ldots, X_{n}\right\}, i=1,2, \ldots, n$.

(iii) $\mathrm{Li}$ and $\mathrm{Hu}[5]$ proved Corollary 2.1(iii).

(iv) $\mathrm{Li}$ and $\mathrm{Hu}$ [5] proved that if $X_{1}$ or $X_{2}$ has a convex survival function and $X_{1} \leq_{\text {icv }} X_{2}$, then $\vee\left\{X_{1}+X, X_{2}\right\} \leq_{\text {sp }} \vee\left\{X_{1}, X_{2}+X\right\}$. Corollary 2.1(iv) may be viewed as a supplement of the result proved in [5].

\section{Allocation of two standby redundancies}

In this section we deal with stochastic comparisons between $Z_{1}$ and $Z_{2}$ (defined by (1.1)) and also between $Z_{1}^{\prime}$ and $Z_{2}^{\prime}$ (defined by (1.2)). Let $\bar{H}$ and $h$ respectively denote the survival function and the Lebesgue density function of $T=\wedge\left\{X_{3}, X_{4}, \ldots, X_{n}\right\}$. Then

$$
h(t)=\sum_{i=3}^{n} f_{i}(t) \prod_{\substack{j=3 \\ j \neq i}}^{n} \bar{F}_{j}(t), \quad t \in \mathbb{R}_{+} .
$$

Theorem 3.1. Suppose that $X_{1} \leq_{\mathrm{icv}} X_{2}, Y_{1} \leq_{\mathrm{hr}} Y_{2}$, and that $X_{1}, X_{3}, X_{4}, \ldots, X_{n}$ (or $X_{2}, X_{3}$, $X_{4}, \ldots, X_{n}$ ) have convex survival functions on $\mathbb{R}_{+}$. Then $Z_{2} \leq_{\text {sp }} Z_{1}$.

Proof. It is easy to verify that

$$
\left[Z_{1}>Z_{2}\right]=\left[X_{1}>X_{2}, Y_{1}>Y_{2}, T>X_{2}+Y_{2}\right] \cup\left[X_{2}>X_{1}, Y_{2}>Y_{1}, T>X_{1}+Y_{1}\right] .
$$

Using symmetry, we can write

$$
\begin{aligned}
\Delta_{6} \equiv & \mathrm{P}\left(Z_{1}>Z_{2}\right)-\mathrm{P}\left(Z_{2}>Z_{1}\right) \\
= & \mathrm{P}\left(X_{1}>X_{2}, Y_{1}>Y_{2}, T>X_{2}+Y_{2}\right)+\mathrm{P}\left(X_{2}>X_{1}, Y_{2}>Y_{1}, T>X_{1}+Y_{1}\right) \\
& -\mathrm{P}\left(X_{1}>X_{2}, Y_{2}>Y_{1}, T>X_{2}+Y_{1}\right)-\mathrm{P}\left(X_{2}>X_{1}, Y_{1}>Y_{2}, T>X_{1}+Y_{2}\right) \\
= & \mathrm{E}\left[\int_{0}^{\infty}\left[\bar{G}_{2}(y) g_{1}(y)-\bar{G}_{1}(y) g_{2}(y)\right]\right. \\
& \left.\times\left[\bar{H}\left(X_{1}+y\right) I\left(X_{2}>X_{1}\right)-\bar{H}\left(X_{2}+y\right) I\left(X_{1}>X_{2}\right)\right] \mathrm{d} y\right] .
\end{aligned}
$$


Case I: $X_{1}, X_{3}, X_{4}, \ldots, X_{n}$ have convex survival functions. Let $\hat{X}_{1}$ be an independent copy of $X_{1}$. Then, using (3.2), we can write $\Delta_{6}=\mathrm{E}\left[\psi_{7}\left(X_{2}\right)\right]$, where

$$
\begin{aligned}
\psi_{7}(t)=\mathrm{E}\left[\int_{0}^{\infty}\right. & {\left[\bar{G}_{2}(y) g_{1}(y)-\bar{G}_{1}(y) g_{2}(y)\right] } \\
& \left.\times\left[\bar{H}\left(\hat{X}_{1}+y\right) I\left(t>\hat{X}_{1}\right)-\bar{H}(t+y) I\left(\hat{X}_{1}>t\right)\right] \mathrm{d} y\right], \quad t \in \mathbb{R}_{+} .
\end{aligned}
$$

Obviously, $\hat{X}_{1} \leq_{\mathrm{icv}} X_{2}, \mathrm{E}\left[\psi_{7}\left(\hat{X}_{1}\right)\right]=0$, and

$$
\begin{aligned}
\psi_{7}(t)= & \int_{0}^{t} \int_{0}^{\infty}\left[\bar{G}_{2}(y) g_{1}(y)-\bar{G}_{1}(y) g_{2}(y)\right] \bar{H}(s+y) f_{1}(s) \mathrm{d} y \mathrm{~d} s \\
& -\int_{0}^{\infty}\left[\bar{G}_{2}(y) g_{1}(y)-\bar{G}_{1}(y) g_{2}(y)\right] \bar{H}(t+y) \bar{F}_{1}(t) \mathrm{d} y, \quad t \in \mathbb{R}_{+} .
\end{aligned}
$$

The assumption that $Y_{1} \leq$ hr $Y_{2}$ (i.e. $\bar{G}_{2}(y) g_{1}(y) \geq \bar{G}_{1}(y) g_{2}(y)$ for all $\left.y \in \mathbb{R}_{+}\right)$implies that $\psi_{7}(t)$ is increasing in $t \in \mathbb{R}_{+}$. Since $X_{1}, X_{3}, X_{4}, \ldots, X_{n}$ have convex survival functions on $\mathbb{R}_{+}$(i.e. $f_{i}(\cdot), i=1,3,4, \ldots, n$, are decreasing on $\mathbb{R}_{+}$), it can be easily verified that $\psi_{7}^{\prime}(t)$ is decreasing in $t \in \mathbb{R}_{+}$. Thus, $\psi_{7}(\cdot)$ is an increasing concave function on $\mathbb{R}_{+}$. Now, on using $\hat{X}_{1} \leq_{\text {icv }} X_{2}$, it follows that $\Delta_{6}=\mathrm{E}\left[\psi_{7}\left(X_{2}\right)\right] \geq \mathrm{E}\left[\psi_{7}\left(\hat{X}_{1}\right)\right]=0$, i.e. $Z_{2} \leq_{\text {sp }} Z_{1}$.

Case II: $X_{2}, X_{3}, X_{4}, \ldots, X_{n}$ have convex survival functions. Let $\hat{X}_{2}$ be an independent copy of $X_{2}$. Then we can write $\Delta_{6}=-\mathrm{E}\left[\psi_{8}\left(X_{1}\right)\right]$, where

$$
\begin{aligned}
\psi_{8}(t)=\mathrm{E}\left[\int_{0}^{\infty}[\right. & \left.\bar{G}_{2}(y) g_{1}(y)-\bar{G}_{1}(y) g_{2}(y)\right] \\
& \left.\times\left[\bar{H}\left(\hat{X}_{2}+y\right) I\left(t>\hat{X}_{2}\right)-\bar{H}(t+y) I\left(\hat{X}_{2}>t\right)\right] \mathrm{d} y\right], \quad t \in \mathbb{R}_{+} .
\end{aligned}
$$

Clearly, $X_{1} \leq_{\text {icv }} \hat{X}_{2}$ and $\mathrm{E}\left[\psi_{8}\left(\hat{X}_{2}\right)\right]=0$. Proceeding as in case I, we can show that $\Delta_{6}=$ $-\mathrm{E}\left[\psi_{8}\left(X_{1}\right)\right] \geq-\mathrm{E}\left[\psi_{8}\left(\hat{X}_{2}\right)\right]=0$, i.e. $Z_{2} \leq_{\text {sp }} Z_{1}$.

Theorem 3.2. Suppose that $X_{1} \leq_{\mathrm{icv}} X_{2}, Y_{1} \leq_{\mathrm{st}} Y_{2}, X_{1}$ or $X_{2}$ has convex survival function on $\mathbb{R}_{+}$, and that $X_{i}, i=3,4, \ldots, n$, has a log-convex density on $\mathbb{R}_{+}$. Then $Z_{2} \leq_{\text {sp }} Z_{1}$.

Proof. We will follow the line of the proof of Theorem 3.1. From case I (when $X_{1}$ has convex survival function), we have

$$
\begin{aligned}
\psi_{7}^{\prime}(t) & =\int_{0}^{\infty}\left[\bar{G}_{2}(y) g_{1}(y)-\bar{G}_{1}(y) g_{2}(y)\right]\left[2 \bar{H}(t+y) f_{1}(t)+\bar{F}_{1}(t) h(t+y)\right] \mathrm{d} y \\
& =\mathrm{E}\left[\bar{G}_{2}\left(Y_{1}\right) \varphi\left(t, Y_{1}\right)\right]-\mathrm{E}\left[\bar{G}_{1}\left(Y_{2}\right) \varphi\left(t, Y_{2}\right)\right], \quad t \in \mathbb{R}_{+},
\end{aligned}
$$

where

$$
\varphi(t, y)=2 \bar{H}(t+y) f_{1}(t)+\bar{F}_{1}(t) h(t+y), \quad t, y \in \mathbb{R}_{+} .
$$

Log-convexity of $f_{i}(\cdot)$ implies log-convexity of $\bar{F}_{i}(\cdot), i=3, \ldots, n$ (see Proposition B.8 of [6, p. 101]). Since the product of log-convex functions is log-convex and log-convexity implies convexity, it follows that $\bar{H}(\cdot)$ is convex on $\mathbb{R}_{+}$. Using the abovementioned observations along with the property that the sum of convex functions is convex, we conclude (using (3.1)) that $h(\cdot)$ is a decreasing and convex function on $\mathbb{R}_{+}$. Therefore, for each fixed $t \in \mathbb{R}_{+}, \bar{G}_{2}(y) \varphi(t, y)$ is decreasing in $y \in \mathbb{R}_{+}$. Now, using $Y_{1} \leq_{\text {st }} Y_{2}$, we obtain

$$
\mathrm{E}\left[\bar{G}_{2}\left(Y_{1}\right) \varphi\left(t, Y_{1}\right)\right] \geq \mathrm{E}\left[\bar{G}_{2}\left(Y_{2}\right) \varphi\left(t, Y_{2}\right)\right] \geq \mathrm{E}\left[\bar{G}_{1}\left(Y_{2}\right) \varphi\left(t, Y_{2}\right)\right],
$$

i.e. $\psi_{7}^{\prime}(t) \geq 0$ for all $t \in \mathbb{R}_{+}$. 
Let $0 \leq t_{1}<t_{2}<\infty$. Consider

$$
\begin{aligned}
\Delta_{7} & =\psi_{7}^{\prime}\left(t_{1}\right)-\psi_{7}^{\prime}\left(t_{2}\right) \\
& =\mathrm{E}\left[\bar{G}_{2}\left(Y_{1}\right)\left\{\varphi\left(t_{1}, Y_{1}\right)-\varphi\left(t_{2}, Y_{1}\right)\right\}\right]-\mathrm{E}\left[\bar{G}_{1}\left(Y_{2}\right)\left\{\varphi\left(t_{1}, Y_{2}\right)-\varphi\left(t_{2}, Y_{2}\right)\right\}\right] .
\end{aligned}
$$

Convexity of $\bar{H}(\cdot)$ and $h(\cdot)$ on $\mathbb{R}_{+}$implies that $\bar{H}\left(t_{2}+y_{1}\right)-\bar{H}\left(t_{2}+y_{2}\right) \leq \bar{H}\left(t_{1}+y_{1}\right)-$ $\bar{H}\left(t_{1}+y_{2}\right)$ and $h\left(t_{2}+y_{1}\right)-h\left(t_{2}+y_{2}\right) \leq h\left(t_{1}+y_{1}\right)-h\left(t_{1}+y_{2}\right)$ whenever $0 \leq t_{1}<t_{2}<\infty$ and $0 \leq y_{1}<y_{2}<\infty$. Also, convexity of the survival function of $X_{1}$ implies that $f_{1}(\cdot)$, and, hence, $\varphi(\cdot, y)$, is decreasing on $\mathbb{R}_{+}$. Using these observations, it can be verified that, for each fixed $0 \leq t_{1}<t_{2}<\infty, \varphi\left(t_{1}, y\right)-\varphi\left(t_{2}, y\right)$ (and, hence, $\bar{G}_{2}(y)\left\{\varphi\left(t_{1}, y\right)-\varphi\left(t_{2}, y\right)\right\}$ ) is decreasing in $y \in \mathbb{R}_{+}$, i.e. $\varphi\left(t_{1}, y_{1}\right)-\varphi\left(t_{2}, y_{1}\right) \geq \varphi\left(t_{1}, y_{2}\right)-\varphi\left(t_{2}, y_{2}\right)$ whenever $0 \leq y_{1}<y_{2}<\infty$. Now, using $Y_{1} \leq_{\text {st }} Y_{2}$, we obtain

$$
\begin{aligned}
\mathrm{E}\left[\bar{G}_{2}\left(Y_{1}\right)\left\{\varphi\left(t_{1}, Y_{1}\right)-\varphi\left(t_{2}, Y_{1}\right)\right\}\right] & \geq \mathrm{E}\left[\bar{G}_{2}\left(Y_{2}\right)\left\{\varphi\left(t_{1}, Y_{2}\right)-\varphi\left(t_{2}, Y_{2}\right)\right\}\right] \\
& \geq \mathrm{E}\left[\bar{G}_{1}\left(Y_{2}\right)\left\{\varphi\left(t_{1}, Y_{2}\right)-\varphi\left(t_{2}, Y_{2}\right)\right\}\right],
\end{aligned}
$$

i.e. $\Delta_{7} \geq 0$. Thus, $\psi_{7}(\cdot)$ is an increasing concave function on $\mathbb{R}_{+}$. Since $\hat{X}_{1} \leq_{\text {icv }} X_{2}$, we obtain $\Delta_{6}=\mathrm{E}\left[\psi_{7}\left(X_{2}\right)\right] \geq \mathrm{E}\left[\psi_{7}\left(\hat{X}_{1}\right)\right]=0$, i.e. $Z_{2} \leq_{\text {sp }} Z_{1}$.

Using similar techniques, we can show that $Z_{2} \leq_{\text {sp }} Z_{1}$ holds for case II (when $X_{2}$ has convex survival function).

Theorem 3.3. Suppose that $X_{1} \leq_{\mathrm{icx}} X_{2}, Y_{1} \leq_{\mathrm{rh}} Y_{2}$, and that $X_{1}, X_{3}, X_{4}, \ldots, X_{n}\left(\right.$ or $X_{2}, X_{3}$, $X_{4}, \ldots, X_{n}$ ) have concave survival functions on $\mathbb{R}_{+}$. Then $Z_{1}^{\prime} \leq_{\mathrm{sp}} Z_{2}^{\prime}$.

Proof. We will prove the result for the case when $X_{1}, X_{3}, X_{4}, \ldots, X_{n}$ have concave survival functions on $\mathbb{R}_{+}$. The proof for the other case follows along similar lines. Let $T_{1}=$ $\vee\left\{X_{3}, X_{4}, \ldots, X_{n}\right\}$. Then it can be easily verified that

$$
\left[Z_{2}^{\prime}>Z_{1}^{\prime}\right]=\left[X_{1}>X_{2}, Y_{1}>Y_{2}, X_{1}+Y_{1}>T_{1}\right] \cup\left[X_{2}>X_{1}, Y_{2}>Y_{1}, X_{2}+Y_{2}>T_{1}\right] .
$$

Let $K$ be the distribution function of $T_{1}$. Using symmetry, we obtain

$$
\begin{aligned}
\Delta_{8}= & \mathrm{P}\left(Z_{2}^{\prime}>Z_{1}^{\prime}\right)-\mathrm{P}\left(Z_{1}^{\prime}>Z_{2}^{\prime}\right) \\
= & \mathrm{P}\left(X_{1}>X_{2}, Y_{1}>Y_{2}, X_{1}+Y_{1}>T_{1}\right)+\mathrm{P}\left(X_{2}>X_{1}, Y_{2}>Y_{1}, X_{2}+Y_{2}>T_{1}\right) \\
& -\mathrm{P}\left(X_{1}>X_{2}, Y_{2}>Y_{1}, X_{1}+Y_{2}>T_{1}\right)-\mathrm{P}\left(X_{2}>X_{1}, Y_{1}>Y_{2}, X_{2}+Y_{1}>T_{1}\right) \\
= & \mathrm{E}\left[\int_{0}^{\infty}\left[G_{2}(y) g_{1}(y)-G_{1}(y) g_{2}(y)\right]\right. \\
& \left.\quad \times\left[K\left(X_{1}+y\right) I\left(X_{1}>X_{2}\right)-K\left(X_{2}+y\right) I\left(X_{2}>X_{1}\right)\right] \mathrm{d} y\right] \\
& \quad \mathrm{E}\left[\psi_{9}\left(X_{2}\right)\right],
\end{aligned}
$$

where

$$
\begin{aligned}
\psi_{9}(t)=\mathrm{E}\left[\int_{0}^{\infty}\right. & {\left[G_{2}(y) g_{1}(y)-G_{1}(y) g_{2}(y)\right] } \\
& \left.\times\left[K\left(\hat{X}_{1}+y\right) I\left(\hat{X}_{1}>t\right)-K(t+y) I\left(t>\hat{X}_{1}\right)\right] \mathrm{d} y\right], \quad t \in \mathbb{R}_{+},
\end{aligned}
$$


and $\hat{X}_{1}$ is an independent copy of $X_{1}$. Evidently, $\hat{X}_{1} \leq_{\text {icx }} X_{2}$ and $\mathrm{E}\left[\psi_{9}\left(\hat{X}_{1}\right)\right]=0$. From (3.3) we have

$$
\begin{aligned}
\psi_{9}(t)= & \int_{t}^{\infty} \int_{0}^{\infty}\left[G_{2}(y) g_{1}(y)-G_{1}(y) g_{2}(y)\right] K(s+y) f_{1}(s) \mathrm{d} y \mathrm{~d} s \\
& -\int_{0}^{\infty}\left[G_{2}(y) g_{1}(y)-G_{1}(y) g_{2}(y)\right] K(t+y) F_{1}(t) \mathrm{d} y, \quad t \in \mathbb{R}_{+} .
\end{aligned}
$$

Using $Y_{1} \leq_{\text {rh }} Y_{2}$ and the concavity of the survival functions of $X_{1}, X_{3}, X_{4}, \ldots, X_{n}$, it can be verified that $\psi_{9}(\cdot)$ is an increasing convex function on $\mathbb{R}_{+}$. Now, using $\hat{X}_{1} \leq_{\text {icx }} X_{2}$, we obtain $\Delta_{8}=\mathrm{E}\left[\psi_{9}\left(X_{2}\right)\right] \geq \mathrm{E}\left[\psi_{9}\left(\hat{X}_{1}\right)\right]=0$, i.e. $Z_{1}^{\prime} \leq_{\mathrm{sp}} Z_{2}^{\prime}$.

\section{Acknowledgements}

The authors are thankful to the anonymous referee for his/her valuable comments. The second author would like to acknowledge financial assistance from C.S.I.R., India, for carrying out this research work.

\section{References}

[1] Barlow, R. E. And Proschan, F. (1975). Statistical Theory of Reliability and Life Testing. Holt, Rinehart and Winston, New York.

[2] Boland, P. J., El-Neweini, E. And Proschan, F. (1992). Stochastic order for redundancy allocations in series and parallel systems. Adv. Appl. Prob. 24, 161-171.

[3] Boland, P. J., Proschan, F. And Tong, Y. L. (1991). Standby redundancy policies for series systems. Tech. Rep. M-838, Florida State University.

[4] LAI, C. D. ANd XIE, M. (2006). Stochastic Ageing and Dependence for Reliability. Springer, New York.

[5] Li, X. AND Hu, X. (2008). Some new stochastic comparisons for redundancy allocations in series and parallel systems. Statist. Prob. Lett. 78, 3388-3394.

[6] Marshall, A. W. and Olkin, I. (2007). Life Distributions. Springer, New York.

[7] Mi, J. (1998). Bolstering components for maximizing system lifetime. Naval Res. Logistics 45, 497-509.

[8] Müller, A. and Stoyan, D. (2002). Comparison Methods for Stochastic Models and Risks. John Wiley, Chichester.

[9] Romera, R., Valdés, J. E. And Zequeira, R. I. (2004). Active-redundancy allocation in systems. IEEE Trans. Reliab. 53, 313-318.

[10] Shaked, M. and Shanthikumar, J. G. (1992). Optimal allocation of resources to nodes of parallel and series systems. Adv. Appl. Prob. 24, 894-914.

[11] Shaked, M. and Shanthikumar, J. G. (2007). Stochastic Orders. Springer, New York.

[12] Singh, H. AND Misra, N. (1994). On redundancy allocations in systems. J. Appl. Prob. 31, 1004-1014.

[13] Valdés, J. E. AND Zequeira, R. I. (2003). On the optimal allocation of an active redundancy in a two-component series system. Statist. Prob. Lett. 63, 325-332.

[14] Valdés, J. E. AND Zequeira, R. I. (2006). On the optimal allocation of two active redundancies in a twocomponent series system. Operat. Res. Lett. 34, 49-52. 